\title{
Multifractality and intermediate statistics in quantum maps
}

\author{
J. Martin, O. Giraud, and B. Georgeot \\ Laboratoire de Physique Théorique, Université de Toulouse, UPS, CNRS, 31062 Toulouse France \\ (Received 13 November 2007; revised manuscript received 15 January 2008; published 14 March 2008)

\begin{abstract}
We study multifractal properties of wave functions for a one-parameter family of quantum maps displaying the whole range of spectral statistics intermediate between integrable and chaotic statistics. We perform extensive numerical computations and provide analytical arguments showing that the generalized fractal dimensions are directly related to the parameter of the underlying classical map, and thus to other properties such as spectral statistics. Our results could be relevant for Anderson and quantum Hall transitions, where wave functions also show multifractality.
\end{abstract}

DOI: 10.1103/PhysRevE.77.035201

PACS number(s): 05.45.Mt, 05.40.-a, 05.45.Df, 71.30.+h

Statistical properties of wave functions and energy levels of quantum systems have been an important topic of research in the past decades with many applications to physical systems. It is now well known that energy levels of, e.g., quantum systems whose classical limit is chaotic, or disordered systems when eigenstates are extended, follow random matrix theory (RMT). In this case, wave functions are typically ergodic and level statistics show level repulsion at short distances. Conversely, systems whose classical limit is integrable, or disordered systems in a regime of Anderson localization, show Poisson statistics of energy levels (without level repulsion), and wave functions are typically localized in phase space [1].

It was realized later that another universality class exists which is intermediate between the latter two. It can be observed in disordered systems at the Anderson transition [2], or in certain systems whose classical limit is pseudointegrable [3]. In this case, level statistics follow specific laws called semi-Poisson statistics, and wave functions generally show multifractal properties. This multifractal behavior has been extensively studied in the case of the Anderson transition [4-6], and has also been seen in quantum Hall transitions [7]. Multifractality or fractality has also been observed in some other intermediate systems [8], in eigenstates of certain chaotic systems [9], and in open systems [10].

Recently, a simple model for intermediate systems was introduced which corresponds to a quantization of a certain interval-exchange map [11]. The model, although very simple, displays the whole range of semi-Poisson statistics when a parameter is changed. Moreover, a certain randomization of this system yields a new model of random matrices with intermediate statistics [12].

Here, we examine multifractal properties of eigenfunctions for the random matrix model corresponding to intermediate quantum maps. We compute the inverse participation ratios (IPRs), fractal dimensions, and singularity spectra in a variety of regimes with different numerical methods. Using extensive numerical studies and analytical arguments, we show that the parameter of the model can be related to the fractal dimensions of the eigenfunctions, as well as to the spectral statistics. This random matrix model is known to span the whole range of semi-Poisson statistics for both short-range and long-range statistics. Thus our results indicate with some generality the existence of a link between statistics of eigenvalues and multifractal properties of eigenfunctions.
The random matrix model we study is constructed from an intermediate quantum map, which is the quantization of the classical map defined on the two-torus by $\Phi_{\gamma}: \bar{p}=p+\gamma$ $(\bmod 1) ; \bar{q}=q+2 \bar{p}(\bmod 1)$, where $(p, q)$, the coordinates in phase space, are the conjugated action and angle variables and the bars denote the resulting variables after one iteration of the map. Periodic orbits appear in families corresponding to structures more complicated than tori, as in pseudointegrable systems. The quantization of this map yields a unitary evolution operator acting on a Hilbert space of dimension $N=1 /(2 \pi \hbar)$ which can be expressed in momentum space by the $N \times N$ matrix $[11,12]$

$$
U_{p p^{\prime}}=\frac{e^{i \phi_{p}}}{N} \frac{1-e^{2 i \pi N \gamma}}{1-e^{2 i \pi\left(p-p^{\prime}+N \gamma\right) / N}},
$$

with $\phi_{p}=-2 \pi p^{2} / N$. From this quantized map one can construct an ensemble of random matrices, taking $\phi_{p}$ as random variables uniformly distributed in $[0,2 \pi[[12]$. The statistical properties of the pseudospectrum (the set of eigenphases) of $U$ strongly depend on the value of the parameter $\gamma$. On the one hand, for generic irrational $\gamma$, the spectral statistics of $U$ are expected to follow those of the circular unitary ensemble (CUE) of RMT if the $\phi_{p}$ are independent (nonsymmetric case), or the circular orthogonal ensemble (COE) if one imposes a symmetry $\phi_{N-p}=\phi_{p}$. On the other hand, for rational $\gamma=a / b$, a variety of different behaviors are observed [11]. It was shown in Ref. [12] that for $a N= \pm 1 \bmod b$ the spectral statistics is of semi-Poisson type. In particular the nearest-neighbor spacing distribution is given by $P_{\beta}(s)=A_{\beta} s^{\beta} e^{-(\beta+1) s}$ with parameter $\beta=b-1$ in the nonsymmetric case $(\beta=b / 2-1$ in the symmetric case). For $a N \neq \pm 1 \bmod b, P(s)$ is still of intermediate type. Finally when $\gamma$ is an integer the eigenphases are equally spaced and the spectrum is totally rigid. Thus the set of quantum maps $U$ with rational $\gamma$ gives a random matrix ensemble with intermediate statistics (ISRM) whose spectral statistics correspond to natural intermediate distributions between Poisson and RMT, controlled by the value of $\gamma$.

Multifractality properties of wave functions are described by a whole set of generalized fractal dimensions $D_{q}$. For a vector $|\psi\rangle=\sum_{i=1}^{N} \psi_{i}|i\rangle$ in an $N$-dimensional Hilbert space, the multifractal exponents $D_{q}$ are defined through the scaling of the moments 


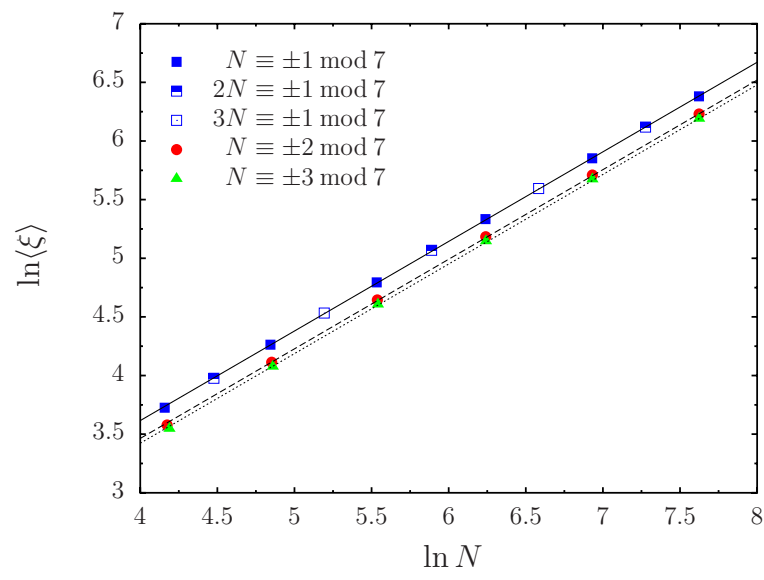

FIG. 1. (Color online) Mean IPR of eigenvectors of Eq. (1) as a function of the vector size $N$ for $\gamma=a / b$ with $b=7$ and $a=1$ (filled symbols), $a=2$ (half-filled squares), $a=3$ (empty squares). Straight lines correspond to the best linear fits. The logarithm is natural.

$$
\sum_{i=1}^{N}\left|\psi_{i}\right|^{2 q} \propto N^{-D_{q}(q-1)} .
$$

The fractal dimension for $q=0$ corresponds to the dimension of the support of the measure, which here is $D_{0}=1$. The fractal exponent $D_{2}$ describes the large-size behavior of the IPR $\xi=1 / \Sigma_{i=1}^{N}\left|\psi_{i}\right|^{4}$, which measures the extension of the state $|\psi\rangle$ over the basis vectors. The multifractal exponents describe the behavior of the partition function

$$
Z(q, L) \equiv \sum_{k=1}^{N_{b}} \mu_{k}(L)^{q} \propto L^{\tau_{q},} \quad \tau_{q} \equiv D_{q}(q-1),
$$

where the vector $|\psi\rangle$ is divided into $N_{b}=N / L$ boxes $B_{k}$ of size $L$, and $\mu_{k}(L)=\sum_{i \in B_{k}}\left|\psi_{i}\right|^{2}, 1 \leqslant k \leqslant N_{b}$. The multifractal properties are alternatively characterized by the singularity spectrum $f(\alpha)$, which is the fractal dimension of the set of points whose singularity exponent is $\alpha$. It is related to the function $\tau_{q}$ by a Legendre transform. Introducing the normalized measures $\mu_{k}(q, L)=\mu_{k}(L)^{q} / \sum_{i} \mu_{i}(L)^{q}$, the singularity exponent and the associated fractal dimension can, respectively, be obtained by [13]

$$
\begin{gathered}
\alpha(q)=\frac{d \tau_{q}}{d q}=\lim _{L / N \rightarrow 0} \frac{\sum_{i} \mu_{i}(q, L) \ln \mu_{i}(L)}{\ln (L / N)}, \\
f[\alpha(q)]=q \alpha(q)-\tau_{q}=\lim _{L / N \rightarrow 0} \frac{\sum_{i} \mu_{i}(q, L) \ln \mu_{i}(q, L)}{\ln (L / N)} .
\end{gathered}
$$

Let us consider an ensemble of matrices of type (1) with rational $\gamma=a / b$, in the nonsymmetric case where all $\phi_{p}$ are independent random variables. The mean IPR for eigenvectors of these matrices in $p$ representation for different values of $\gamma$ with denominator $b=7$ is displayed in Fig. 1 as a function of the matrix size. The IPR scales as $N^{D_{2}}$ provided data corresponding to different values of $a N \bmod b$ be treated separately. Indeed, when different matrix sizes are grouped into families, the results yield a linear behavior of $\ln \langle\xi\rangle$ as a

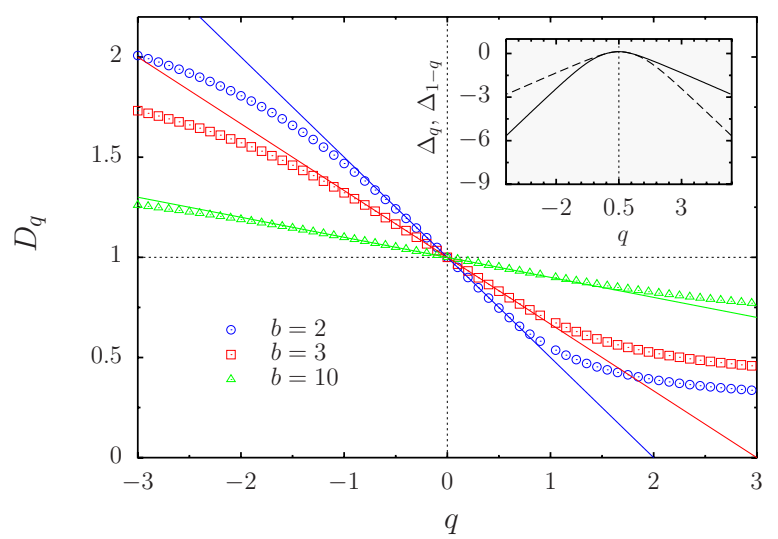

FIG. 2. (Color online) Fractal dimension $D_{q}$ computed with the canonical method for $\gamma=1 / b$ with $b=2$ (blue circles), $b=3$ (red squares), $b=10$ (green triangles). Solid lines show the linear approximation $D_{q}=1-q / b$. Inset: anomalous exponent $\Delta_{q}$ as a function of $q$ (solid line), together with its symmetry with respect to $q=1 / 2$ (dashed line) for $b=2$.

function of $\ln N$, with the same slope $D_{2}$ for each family. More generally we observed that the fractal exponents $D_{q}$ are well defined if data are organized into families, and that they only depend on the denominator $b$ of $\gamma$.

We now proceed to compute the fractal exponents $D_{q}$. A few of these exponents have already been computed in Refs. $[12,14]$ for the case $a N \equiv \pm 1 \bmod b$. Here our aim is to characterize $D_{q}$ as a function of $q$. The quantities $D_{q}$ and $f(\alpha)$ are known to be difficult to compute numerically, especially for large $q$ or $\alpha$. In this work, we resorted to several different methods as a consistency check. We first opted for the usual method of moments. We computed average values of the moments (2) for different system sizes $N$ ranging from $\sim 2000$ up to $\sim 12000$ to get rid as much as possible of finite size effects [15]. The fractal dimensions are extracted from the slopes of the graphs of $\ln \left\langle\Sigma_{i}\left|\psi_{i}\right|^{2 q}\right\rangle$ versus $\ln N$. Here the average is taken over all eigenvectors and random realizations of $U$ (from 200 realizations for $N \sim 2000$ to 1 for $N \sim 12000)$. We also opted for the so-called canonical method [13] allowing one to determine the $f(\alpha)$ spectrum directly from Eq. (4). For this method, the numerical computations were done on a single realization of size $N$ $\sim 13000$, and 20 box sizes ranging from $L=10$ to $L=0.1 \mathrm{~N}$ (again different families of box sizes were treated separately). We also considered other approaches, such as the box counting method based on Eq. (3); they all give results intermediate between the two previous methods.

The results for $D_{q}$ are displayed in Fig. 2. For increasing $b$, the curve for $D_{q}$ tends slowly to the limiting curve where $D_{q}=1$ for all $q$, which corresponds to nonfractal wave functions. This is in agreement with the fact that $\gamma$ tends for $b \rightarrow \infty$ to an irrational number for which the system is expected to follow RMT. Figure 2 shows that $D_{q}$ is roughly linear in a relatively large interval around $q=0$, and tends to limiting values $D_{ \pm \infty}$ for large $|q|$. The slope of $D_{q}$ at $q=0$ is displayed in the inset of Fig. 3 as a function of $b$. We found that the value of this slope is very accurately given by $-1 / b$. Since $D_{0}=1$, the first-order expansion of $D_{q}$ around $q=0$ reads 


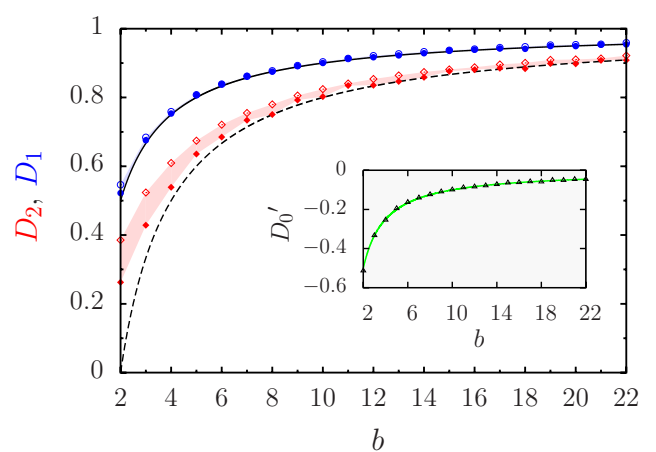

FIG. 3. (Color online) Information dimension $D_{1}$ (blue circles) and correlation dimension $D_{2}$ (red diamonds) as a function of the denominator of $\gamma=1 / b$. Full (empty) symbols are numerical values obtained by the method of moments (the canonical method). Solid and dashed lines are the theoretical curves $1-1 / b$ and $1-2 / b$ for $D_{1}$ and $D_{2}$, respectively. The values obtained by, e.g., the box counting method lie in the shaded domain in between. Inset: slope of $D_{q}$ at the origin $q=0$ (triangles) and curve $-1 / b$ (solid line).

$$
D_{q} \approx 1-\frac{q}{b}
$$

This expansion turns out to be valid in a quite large interval of $q$, whose size increases with $b$. As an example, the numerical values of $D_{1}$ and $D_{2}$ together with the linear approximation (5) are shown in Fig. 3. When $b$ is increased, Eq. (5) is verified with higher and higher accuracy. Of course Eq. (5) breaks down for large $|q|$ since $D_{q}$ is bounded.

To get an understanding of why formula (5) holds, we note that general arguments for critical systems predict that the multifractal properties of the eigenstates for $q=2$ are linked to the spectral statistics through a relation between the correlation dimension $D_{2}$ and the level compressibility $\chi$ [16],

$$
\chi=\frac{1}{2}\left(1-\frac{D_{2}}{D_{0}}\right)
$$

Numerical results for the power-law random banded matrix ensemble [5] have revealed that this relation is extremely well verified in the regime of weak multifractality (large bands): in this model, the fractal dimension evolves linearly with respect to $q$ as $D_{q}=1-\kappa q$, where $\kappa$ is inversely proportional to the width of the central band (and in this particular case can be also related to the level compressibility). However, for smaller bands Eq. (6) was clearly violated. Suppose Eq. (6) holds in our case. For ISRM [Eq. (1)], the level compressibility can be estimated analytically. It is given by the value of the two-point correlation form factor $K_{2}(n / N)$ $=\left|\operatorname{Tr} U^{n}\right|^{2} / N$ for $n / N \rightarrow 0$. Following Ref. [11], we note that in the semiclassical limit $N \rightarrow \infty$ and fixed $n$, the trace $\operatorname{Tr} U^{n}$ is asymptotically equal to $\operatorname{Tr} V_{n}$, where $V_{n}$ is the quantization of the $n$th iterate of the classical map, and

$$
\operatorname{Tr} V_{n}=\frac{1}{N} \sum_{p=0}^{N-1} \exp \left(i n \phi_{p}\right) \sum_{k=0}^{N-1} \exp (2 i \pi n \gamma k)
$$

The modulus squared of the first sum yields $\approx N$ when all $\phi_{p}$ are random. The second sum is a geometric sum: for $\gamma=a / b$, it is equal to $N$ if $n$ is divisible by $b$ and to $O(1)$ otherwise. Thus, $K_{2}(n / N) \sim 1$ if $n$ is divisible by $b$, $K_{2}(n / N) \sim 0$ in all other cases. The level compressibility is then given by the time averaged form factor

$$
\chi=\overline{K_{2}(0)} \equiv \lim _{n \rightarrow \infty} \lim _{N \rightarrow \infty} \frac{1}{n} \sum_{n^{\prime}=1}^{n} K_{2}\left(n^{\prime} / N\right) \approx \frac{1}{b} .
$$

Inserting this value of $\chi$ into Eq. (6) we get $D_{2} \approx 1-2 / b$, which corresponds to Eq. (5) for $q=2$. A simple linear interpolation between this value for $D_{2}$ and $D_{0}=1$ yields Eq. (5). We note that for small $b$ (strong multifractality) Eq. (6) breaks down but Eq. (5) is still valid for smaller $q$ values.

Before moving to the study of the singularity spectrum, we briefly discuss symmetry properties of $D_{q}$. It was suggested in Ref. [6] that the anomalous exponents $\Delta_{q}$, defined by $\Delta_{q} \equiv\left(D_{q}-1\right)(q-1)$, approximately follow the symmetry relation $\Delta_{q}=\Delta_{1-q}$. This was shown to hold for the Anderson model with good accuracy over a large interval of $q$ values. It is not the case in our system. As an example, the inset in Fig. 2 gives $\Delta_{q}$ and $\Delta_{1-q}$ for $\gamma=1 / 2$. For values of $q$ where the exponents $D_{q}$ have a linear behavior the symmetry relation holds, as it should since any linear $D_{q}$ necessarily fulfills it. Outside the linear regime, the relation is no longer verified.

We now turn to the singularity spectrum $f(\alpha)$. For $\gamma=a / b$, the expression obtained using Eq. (5) is

$$
f(\alpha) \approx 1-\frac{b}{4}\left(\alpha-1-\frac{1}{b}\right)^{2}
$$

It reaches its maximum at $\alpha(q=0)=1+1 / b$. Since Eq. (5) is valid around $q=0$, we expect Eq. (9) to be accurate around $\alpha(0)$. Figure 4 shows the singularity spectrum, numerically computed using Eq. (4), together with the theoretical estimate Eq. (9). The data displayed show that Eq. (9) approximates the singularity spectrum with good accuracy over a large interval of values of $\alpha$ around $\alpha(0)$. As $b \rightarrow \infty$, the curve for $f(\alpha)$ gets closer and closer to a single point at $\alpha=1$, once again corresponding to the nonfractal case of RMT.

We previously showed that the theory (5) accurately describes the behavior of the moments in Eq. (2) for small $q$. For large values of $q$, this is bound to break down since $D_{q}$ converges to a finite asymptotic value for $q \rightarrow \pm \infty$. Similarly $f(\alpha)$ should have vertical asymptotes at some limiting values $\alpha_{\max }$ and $\alpha_{\min }$, while Eq. (9) is the equation of a parabola. However, numerical data are not far from the theory (9), and some of the features of $f(\alpha)$ are well captured by this estimate. For example, the inset of Fig. 4 shows that the width $w=\alpha_{\max }-\alpha_{\min }$ of the singularity spectrum scales as $\sim 1 / b^{0.53}$ (best fit). This is close to the scaling $1 / \sqrt{b}$ of the difference between the two intersections of the parabola (9) with a straight line. 


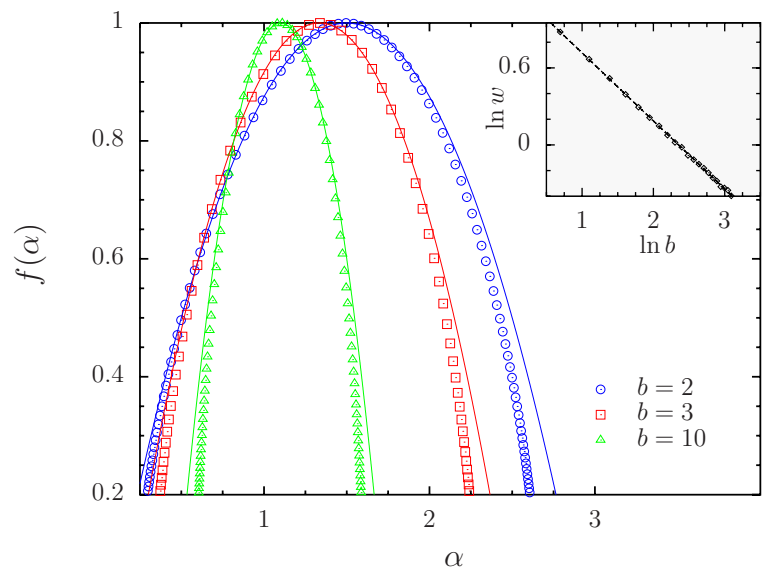

FIG. 4. (Color online) Singularity spectrum $f(\alpha)$ for $\gamma=1 / b$ with $b=2$ (blue circles), $b=3$ (red squares), $b=10$ (green triangles). Solid lines show the parabola (9). Inset: singularity spectrum width $w=\alpha_{\max }-\alpha_{\min }$ as a function of $b$. The dashed line shows the best linear fit $\ln w=-0.530 \ln b+1.247$. The logarithm is natural.

We finish by noting that in the symmetric case $\left[\phi_{N-p}=\phi_{p}\right.$ in Eq. (1)] we have performed similar computations, getting very similar data. In particular, Eqs. (5) and (9) are valid in this case as well.

In conclusion, we have studied multifractal properties of eigenfunctions for intermediate quantum maps. Although data corresponding to system sizes $N$ with different values of $a N \bmod b$ should be treated separately, they give the same value for $D_{q}$ and $f(\alpha)$. Our results show that for an interval of $q$ values whose size increases with $b$, the fractal exponents can be related explicitly to the parameter $\gamma=a / b$ of the map through Eq. (5), and thus to spectral statistics. A similar result holds for the singularity spectrum through Eq. (9). Thus in such a system, fractal exponents and singularity spectrum are related to the spectral properties over a wide range of fractal dimensions. Interestingly enough, our relation is still valid for small $q$ even at values of $b$ where Eq. (6) does not hold. As our system corresponds to a random matrix model covering the whole range of semi-Poisson statistics, both at short range and long range, we can expect our results to display some generality. It will be interesting to study if similar results apply to other intermediate systems, to open systems, and to other physical systems where wave functions are multifractal, such as condensed-matter systems at the Anderson or quantum Hall transitions.

We thank E. B. Bogomolny and K. Frahm for helpful discussions, CalMiP and IDRIS for access to their supercomputers, and the French ANR (Project INFOSYSQQ Contract No. ANR-05-JCJC-0072) and the IST-FET program of the EC (Project EUROSQIP) for funding.
[1] F. Haake, Quantum Signatures of Chaos (Springer, Berlin, 1991).

[2] B. I. Shklovskii, B. Shapiro, B. R. Sears, P. Lambrianides, and H. B. Shore, Phys. Rev. B 47, 11487 (1993); D. Braun, G. Montambaux, and M. Pascaud, Phys. Rev. Lett. 81, 1062 (1998).

[3] E. B. Bogomolny, U. Gerland, and C. Schmit, Phys. Rev. E 59, R1315 (1999); E. Bogomolny, O. Giraud, and C. Schmit, ibid. 65, 056214 (2002).

[4] F. Evers and A. D. Mirlin, Phys. Rev. Lett. 84, 3690 (2000); A. D. Mirlin, Phys. Rep. 326, 259 (2000).

[5] E. Cuevas, M. Ortuno, V. Gasparian, and A. Perez-Garrido, Phys. Rev. Lett. 88, 016401 (2001); A. M. Garcia-Garcia and J. Wang, ibid. 94, 244102 (2005).

[6] A. D. Mirlin, Y. V. Fyodorov, A. Mildenberger, and F. Evers, Phys. Rev. Lett. 97, 046803 (2006).

[7] B. Huckestein, Rev. Mod. Phys. 67, 357 (1995).

[8] A. M. Garcia-Garcia, Phys. Rev. E 72, 066210 (2005).
[9] N. Meenakshisundaram and A. Lakshminarayan, Phys. Rev. E 71, 065303(R) (2005); L. Ermann and M. Saraceno, ibid. 74, 046205 (2006).

[10] G. Casati, G. Maspero, and D. L. Shepelyansky, Physica D 131, 311 (1999); J. P. Keating, M. Novaes, S. D. Prado, and M. Sieber, Phys. Rev. Lett. 97, 150406 (2006).

[11] O. Giraud, J. Marklof, and S. O’Keefe, J. Phys. A 37, L303 (2004).

[12] E. Bogomolny and C. Schmit, Phys. Rev. Lett. 93, 254102 (2004).

[13] A. Chhabra and R. V. Jensen, Phys. Rev. Lett. 62, 1327 (1989)

[14] O. Giraud and B. Georgeot, Phys. Rev. A 72, 042312 (2005).

[15] As $U$ is not defined for $a N=0 \bmod b$, dimensions $N$ are not necessarily the same for all $b$.

[16] J. T. Chalker, V. E. Kravtsov, and I. V. Lerner, JETP Lett. 64, 386 (1996). 